\title{
Infected Pancreatic Hydatid Cyst with Cysto-Pancreatic Fistula Presenting as Acute Pancreatitis
}

\author{
Phillip Daniel ${ }^{1} \quad$ Siddharth Shukla ${ }^{1} \quad$ Nidhi Prabhakar ${ }^{2}$ \\ Rajesh Gupta ${ }^{2}$ Surinder Singh Rana ${ }^{1}$ \\ ${ }^{1}$ Department of Gastroenterology, Post Graduate Institute of \\ Medical Education and Research (PGIMER), Chandigarh, India \\ ${ }^{2}$ Department of Radiodiagnosis, Post Graduate Institute of Medical \\ Education and Research (PGIMER), Chandigarh, India \\ ${ }^{3}$ Department of Surgery, Post Graduate Institute of Medical \\ Education and Research (PGIMER), Chandigarh, India
}

Aditya Kulkarni ${ }^{3} \quad$ Ravi Sharma ${ }^{1}$

\begin{abstract}
Address for correspondence Dr Surinder Singh Rana, MD, DM, Department of Gastroenterology, PGIMER, Chandigarh, 160012 , India (e-mail: drsurinderrana@yahoo.co.in).
\end{abstract}

J Digest Endosc 2020;10:234-236
Abstract
Keywords
- cysts
- endosonography
- hydatid
- pancreatitis

\begin{abstract}
Hydatid cysts can be found in almost any organ of the body with the most common sites being liver, lung, spleen, and kidney. A pancreatic hydatid cyst is rare but an isolated pancreatic hydatid cyst is even rarer. Cysts in the head of pancreas usually present with jaundice, whereas cysts located in the body and tail are usually asymptomatic. We present a rare case of isolated pancreatic hydatid cyst that presented with acute pancreatitis and mimicked a pancreatic fluid collection.
\end{abstract}

\section{Introduction}

Hydatid disease is a zoonotic disease caused by the larval stage of Echinococcus species wherein the dogs are the definitive host and sheep and goats are the intermediate host. ${ }^{1}$ Human acquire infection by accidental ingestion of the Echinococcus eggs in dog feces. These eggs give rise to the larvae of the parasite which migrate from the intestine to the portal circulation. Once in circulation, they can involve any part of the body with liver being the most common site as it is the first organ to filter the portal circulation. Other common sites of involvement are lungs, spleen, and kidney. ${ }^{1,2}$

Pancreatic hydatid cyst is uncommon but isolated pancreatic hydatidosis is even rarer., ${ }^{1,2}$ Cysts in the head of pancreas usually present with jaundice, whereas cysts located in the body and tail are usually asymptomatic. We present a rare case of isolated pancreatic hydatid cyst that presented with acute pancreatitis and mimicked a pancreatic fluid collection.

\section{Case Report}

A 17-year-old girl presented with a 1-month history of epigastric pain associated with high-grade intermittent fever of 3 days duration. The evaluation revealed painful epigastric lump and leukocytosis with elevated serum amylase levels of $768 \mathrm{U} / \mathrm{L}$. Prima facie, a possibility of acute pancreatitis with necrotic collection was considered. Ultrasonography (USG) of the abdomen showed large cystic lesion between the stomach and spleen. Computed tomography (CT) showed a $12 \mathrm{~cm}$ thick-walled cystic lesion containing air in the distal body and tail of the pancreas ( - Fig. 1). Magnetic resonance imaging (MRI) also revealed a large well-defined cystic lesion in the pancreas with multiple membranes typical of hydatid cyst (-Fig. 2).

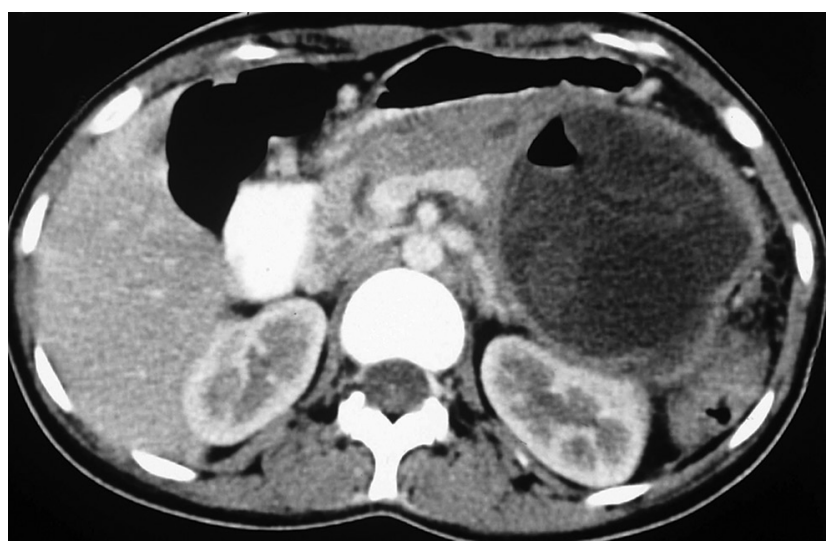

Fig. 1 Computed tomography: $12 \mathrm{~cm}$ thick-walled cystic lesion containing air in distal body and tail of the pancreas.
DOI https://doi.org/

10.1055/s-0040-1708069

ISSN 0976-5042.
(C) 2020 Society of Gastrointestinal Endoscopy of India
License terms

() (1) $\Theta \circledast$ 
Endoscopic ultrasound (EUS) confirmed the presence of a cystic lesion in the distal body and tail of the pancreas. This cystic lesion had multiple daughter cysts sugges-

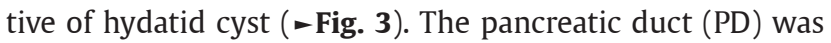
seen communicating with the cystic lesion ( - Video 1). Her hydatid serology was positive, and she was started on oral albendazole and intravenous antibiotics. In view of fever with leukocytosis, endoscopic retrograde pancreatography and drainage were carried out. The ductal communication was confirmed ( $\boldsymbol{- F i g . ~} \mathbf{4}$ and $\boldsymbol{- V}$ Video $\mathbf{1}$ ), and 7Fr transpapillary nasocystic drain was placed. Her fever subsided and follow-up USG revealed reduction in the size of the cyst. Subsequently, she underwent distal pancreatectomy, wedge resection of the adherent gastric wall, and splenectomy (-Fig. 5).

\section{Discussion}

Pancreatic hydatid cysts comprise $<1 \%$ of hydatid cysts and should be considered a differential diagnosis of pancreatic pseudocyst or cystic neoplasm, especially in endemic regions. ${ }^{1}$ However, their rarity is compounded by the overlapping imaging features with other cystic lesions,

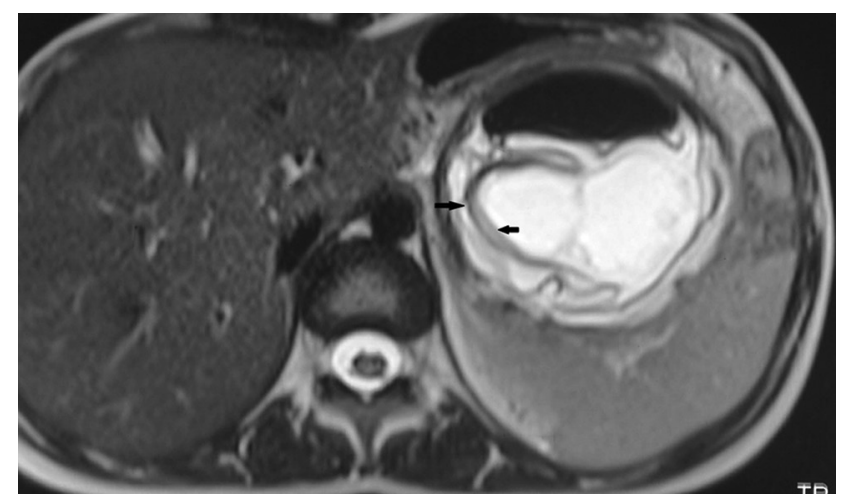

Fig. 2 MRI (axial section): large well-defined cystic lesion in pancreas with multiple membranes (arrow), which is typical of hydatid cyst. Abbreviation: MRI, magnetic resonance imaging.

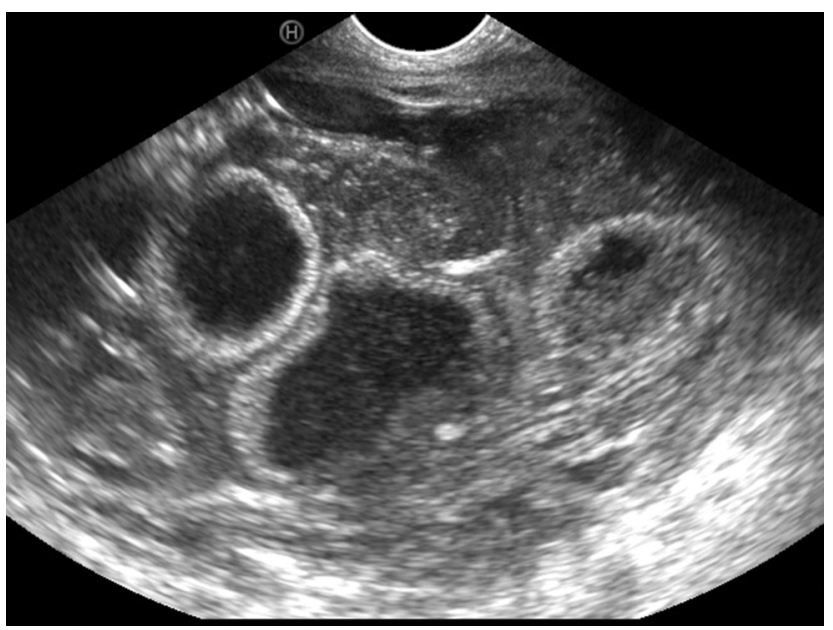

Fig. 3 Endoscopic ultrasound: large cystic lesion in distal body and tail of the pancreas with multiple daughter cysts.

\section{Video 1}

Endoscopic ultrasound showing communication of pancreatic duct with the cyst and endoscopic retrograde cholangiopancreatography, confirming the presence of duct communication by leaking contrast. Online content including video sequences viewable at: https:// www.thieme-connect.com/products/ejournals/html/ 10.1055/s-0040-1708069.

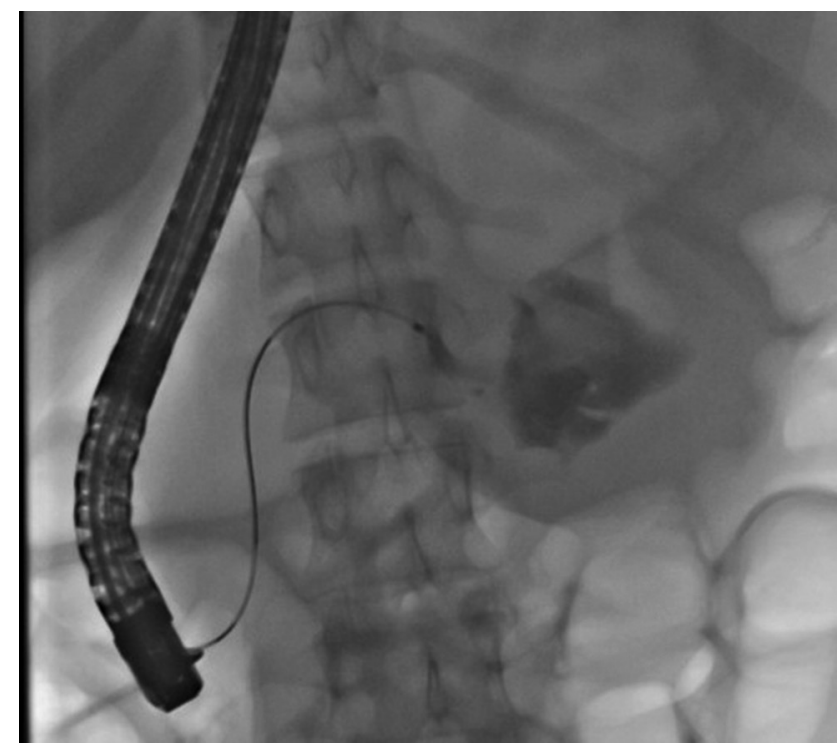

Fig. 4 Endoscopic retrograde pancreatography: contrast leaking from pancreatic duct and filling the cyst cavity, suggestive of duct communication.

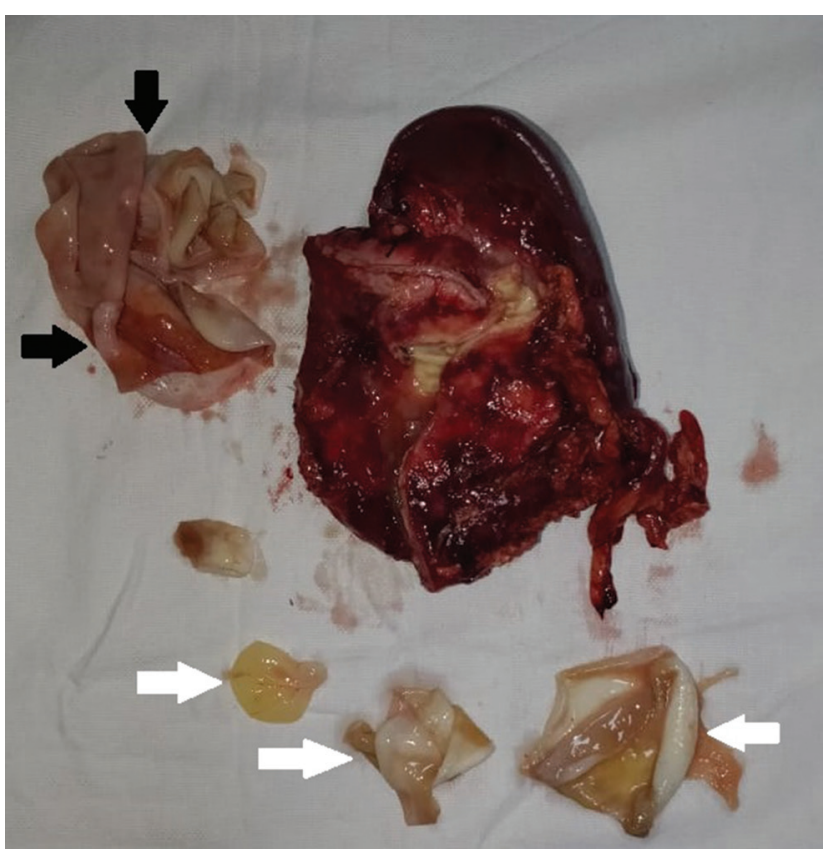

Fig. 5 Surgical specimen: Photograph of cyst cut open showing laminated membrane (black arrow) and daughter cysts (white arrow). 
which makes preoperative diagnosis difficult. These cysts are usually solitary (approximately 90\%) and commonly located in the head of the pancreas. ${ }^{2}$ These cysts may be either primary, involving the pancreas only, or secondary involving other organs, with liver being the most commonly involved. ${ }^{2}$ Blood-borne dissemination is hypothesized to be the most common mode of spread of larva of hydatid cyst to the pancreas. ${ }^{2,3}$ Due to the slow growth, the majority of patients are asymptomatic at the time of detection of the cyst. The symptoms occur because of either fistulization of the cyst into the PD or external compression of the surrounding structures by the enlarged cyst. Cysts in the head of the pancreas usually present with jaundice, whereas cysts located in the body and tail are usually asymptomatic.,3 Cystopancreatic fistulae are very rare, and only four cases were reported in a review of 57 patients. ${ }^{2}$

As for any other pancreatic cyst, the most commonly performed imaging investigations are USG, CT, and MRI.2,3 However, as the pancreas is retroperitoneal structure with visualization impaired by bowel gases, USG plays a limited role in the evaluation of pancreatic cysts. CT helps in accurately delineating the cyst along with its size and presence of cysts in other organs, with MRI and magnetic resonance cholangiopancreatography (MRCP) being useful in delineating the contents of the cyst along with its relationship with pancreatic and bile ducts. However, all these imaging modalities have limited sensitivity in making a specific diagnosis because of considerable overlap of imaging findings. ${ }^{4}$ EUS is a newer imaging modality that provides high-resolution images of the pancreas and is an important investigation procedure for diagnosis and evaluation of pancreatic cystic lesions. ${ }^{5}$ Demonstration of daughter cysts and hydatid sand on EUS can help in the confident diagnosis of pancreatic hydatid disease, as was in the index case. EUS can also help in demonstrating the communication of PD with the cyst. ${ }^{6}$ No single test can help in the accurate diagnosis of pancreatic hydatidosis, and its diagnosis is usually made in an appropriate epidemiological setting, with imaging usually EUS showing daughter cysts and USG, CT or MRI showing an undulating lining membrane along with peripheral eosinophilia and positive hydatid serology. 4,7

Open surgery is the treatment of choice with the type of surgery dependent on the site of the cyst. ${ }^{8}$ Endoscopic retrograde cholangiopancreatography plays no role in either the diagnosis or treatment but may be used for palliation and bridge to surgery in patients with cystopancreatic fistula, leading onto pancreatitis or infection, as was done in the index case.,3 Minimally invasive techniques such as USG-guided percutaneous drainage, using hypertonic (20\%) saline or absolute alcohol as scolicidal agents, have been successfully used for noncommunicating hepatic as well as renal hydatid cysts. ${ }^{910}$ It has also been reported to be successfully used for noncommunicating pancreatic hydatid cysts. ${ }^{11}$ In conclusion, pancreatic hydatid cyst is very rare and should be considered as a differential diagnosis of pancreatic cystic lesions, especially in endemic areas.

\section{Author Contributions}

1. Phillip Daniel: Collection and interpretation of data, drafting of manuscript.

2. Siddharth Shukla: Drafting of manuscript.

3. Nidhi Prabhakar: Collection and interpretation of data.

4. Aditya Kulkarni: Collection and interpretation of data.

5. Ravi Sharma: Collection and interpretation of data.

6. Rajesh Gupta: Collection and interpretation of data.

7. Surinder Singh Rana: Collection and interpretation of data, drafting of manuscript, and conducting critical analysis

\section{Financial Support and Sponsorship}

Nil.

\section{Conflicts of Interest}

There are no conflicts of interest.

\section{References}

1 Dziri C. Hydatid disease-continuing serious public health problem: introduction. World J Surg 2001;25(1):1-3

2 Akbulut S, Yavuz R, Sogutcu N, et al. Hydatid cyst of the pancreas: report of an undiagnosed case of pancreatic hydatid cyst and brief literature review. World J Gastrointest Surg 2014;6(10):190-200

3 Ahmed Z, Chhabra S, Massey A, et al. Primary hydatid cyst of pancreas: case report and review of literature. Int J Surg Case Rep 2016;27:74-77

4 Shah OJ, Robbani I, Zargar SA, et al. Hydatid cyst of the pancreas. An experience with six cases. JOP 2010;11(6):575-581

5 Rana SS, Bhasin DK, Rao C, Gupta R. Intramural pseudocysts of the upper gastrointestinal tract. Endosc Ultrasound 2013;2(4):194-198

6 Diop SP, Costi R, Le Bian A, Carloni A, Meduri B, Smadja C. Acute pancreatitis associated with a pancreatic hydatid cyst: understanding the mechanism by EUS. Gastrointest Endosc 2010;72(6):1312-1314

7 Lemmer ER, Krige JE, Price SK, Girdwood AH. Hydatid cyst in the head of the pancreas with obstructive jaundice. J Clin Gastroenterol 1995;20(2):136-138

8 Dziri C, Dougaz W, Bouasker I. Surgery of the pancreatic cystic echinococcosis: systematic review. Transl Gastroenterol Hepatol 2017;2:105

9 Filice C, Pirola F, Brunetti E, Dughetti S, Strosselli M, Foglieni CS. A new therapeutic approach for hydatid liver cysts. Aspiration and alcohol injection under sonographic guidance. Gastroenterology 1990;98(5 Pt 1) :1366-1368

10 Bastid C, Azar C, Doyer M, Sahel J. Percutaneous treatment of hydatid cysts under sonographic guidance. Dig Dis Sci 1994;39(7):1576-1580

11 Yattoo GN, Khuroo MS, Zargar SA, Bhat FA, Sofi BA. Case report: percutaneous drainage of the pancreatic head hydatid cyst with obstructive jaundice. J Gastroenterol Hepatol 1999;14(9):931-934 